\title{
Inhaled dry salt micro particles in the treatment of bronchopulmonary dysplasia: a five case series report
}

\author{
1 "Carol Davila" University of Medicine and Pharmacy, Bucharest Romania \\ 2 "Marie Curie" Emergency Hospital for Children, Bucharest, Romania
}

NINE LUMINITA², CATALIN G CIRSTOVEANU' ${ }^{2}$ ALEXANDRA I ISTRATE -BARZAN², BARASCU ILEANA $^{2}$, STEFAN MANOLACHE ${ }^{2}$, MIHAELA BIZUBAC ${ }^{2}$, ALINA GAIDUCHEVICI $^{2}$

Corresponding author:

Catalin G Cirstoveanu

"Carol Davila" University of Medicine and Pharmacy

No. 20 Constantin Brancoveanu Boulevard, 4th District

Bucharest, Romania

E-mail: cirstoveanu @yahoo.com

\section{ABSTRACT}

Background. Despite current medical advances, to this day there is no single medical intervention to effectively prevent or treat bronchopulmonary dysplasia (BPD) in both preterm and term infants. Along with protective ventilation strategies, various drugs are being used or are being researched at this very moment, with the sole purpose of improving the general outcome for these patients. Inhaled dry salt micro particles therapy is now one of them.

Materials and methods. This report presents five patients, diagnosed with severe BPD. All of them received, complementary to classical BPD management and respiratory support, continuous inhaled dry salt micro particles, via SaltMed cartridges, for a period of 12 to 30 days. After only 24 hours of administration, we were able to observe a significant improvement in respiratory function and dynamics. It was possible to use a lower fraction of inspired oxygen ( $\mathrm{FiO} 2)$, mean airway pressure (MAP) and peak inspiratory pressure (PIP) in all mechanically ventilated patients. Higher tidal volumes were recorded and we observed improvement in oxygenation indexes.

Conclusion. Continuously inhaled dry salt micro particles, administered complementary to classic BPD management, could improve respiratory and overall morbidity and mortality in infants with any form of BPD. Further study of these possible effects is needed, as there is no data published on this matter so far.

Key words: bronchopulmonary dysplasia (BPD), inhaled dry salt micro particles, oxygenation index (OI), fraction of inspired oxygen (FiO2), mean airway pressure (MAP).

\section{INTRODUCTION}

Bronchopulmonary dysplasia (BPD) remains one of the leading causes for chronic pulmonary disease, in mostly preterm, but also term neonates, that requires prolonged administration of supplemental oxygen and mechanical ventilation.

Constant improvement in neonatal management of these patients, that includes availability of surfactant, the possibility of antenatal glucocorticoid therapy and less invasive mechanical ventilation techniques, has lowered the incidence of the disease and altered its characteristics.

Thus a "new" form of BPD $(1,2)$ is defined, one in which decreased septation and alveolar hypoplasia are the hallmarks, that lead to a smaller gas exchange surface. When accompanied by disruption in vasculogenesis, BPD is often complicated by pulmonary vascular disease, leading to pulmonary hypertension. (2)

A study published by the United States National Institute of Child Health and Human Development (NICHHD), (3) established that the incidence of BPD rises with a decrease in gestational age, with $85 \%$ of the 22 gestational week infants being affected, in comparison to $23 \%$ among those born at 28 weeks of gestational age. There is also a correlation with birth weight: $97 \%$ of cases being diagnosed among infants born with less than 1250 grams. (4)

Commonly cited risk factors for BPD include prematurity, mechanical ventilation and oxygen toxicity, antenatal and postnatal infection, genetic predisposition and possible late surfactant deficiency. (1)

Prevention of BPD is very important as it may lead to the lowering of respiratory morbidity and mortality in these infants. Antenatal glucocorticoid therapy, fluid restriction, protective mechanical ventila- tion strategies, administration of caffeine and possibly supplementation of vitamin A and late surfactant replacement are generally used. $(5,6)$

Management of bronchopulmonary dysplasia is complex and includes respiratory support (mechanical ventilation that uses smaller tidal volumes to avoid further lung tissue trauma and smaller fractions of inspired oxygen in order to lower oxygen toxicity, but enough to ensure adequate tissue oxygenation), nutrition (increased caloric intake alongside often restricted fluid intake) and drug therapy, with a number of single use or combination drugs being used or suggested.

Commonly used drugs are inhaled bronchodilators, used to decrease airway resistance in patients with acute exacerbation of airway obstruction, pulmonary vasodilators (nitric oxide) to reduce pulmonary vascular resistance and inflammation in infants with severe BPD. (7) Systemic and inhaled costicosteroids (8) are being used for their ability to reduce inflammation and promote surfactant production. Diuretics are also commonly used in the management of BPD. Loop diuretics, like Furosemide (intravenous, oral and nebulized), reduce interstitial lung fluid, pulmonary vascular resistance and oxygen requirements. $(7,9)$ They improve short-term lung mechanics, but do not decrease the need for ventilatory support or improve survival. (10)

Unfortunately, despite numerous medical advances, to this day there is no single medical intervention to prevent or treat BPD. A continuous search for new ways of targeted treatment has been launched, including the study of inhaled compounds like heparin, (11) magnesium fluoride and sodium fluoride (MgF, NaF), $(12,13)$ etc., with regards to the possibility of them 


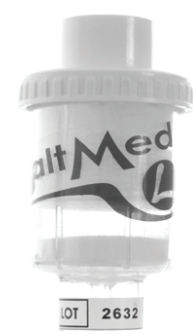

Figure 1. SaltMed dry salt cartridge

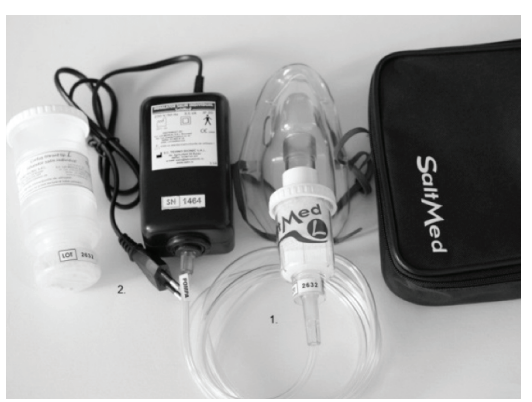

Figure 2. 1/ Dry salt cartridge 2/ Air pump 3/ Cartridge case

tity and clearance through the airways, activating alveolar macrophages and facilitating phagocytosis, bactericide and bacteriostatic effect on respiratory tract microflora. The manufacturer reports that immediate effects of inhaled dry salt micro particles are as follows: increase in quantity of secretions in the upper and lower arespiratory tract, rhinorrhea and sputum, activation of airway cilia and rapid clearance of secretions.

five patients diagnosed with severe BPD, as a complementary intervention in their medical management. To this day there is no published data regarding this kind of therapy in the management of BPD.

The forced passage of air through a specially designed dry salt SaltMed cartridge (figure 1) containing salt microcrystals, produces erosion to a state of micro particles of dry sodium chloride. Their size ranges from 1 to 5 micrometers. This dimension is optimal for the dry salt micro particles to reach and adhere to the respiratory epithelium, where it exerts a therapeutic effect. The air is also filtered and enriched with ions. Total intake of salt per 24 hours of continuous therapy is $3.3 \mathrm{mg}$, as calculated for an adult with an estimated minute volume of $10 \mathrm{l} / \mathrm{min}$. In our five neonates, with an average weight of 3100 grams and with a tidal volume that ranged between 13 and $30 \mathrm{ml}$ (minute volume between 0.4-0.9 $1 / \mathrm{min}$ ), the approximate salt intake would be close to $0.132-0.297 \mathrm{mg}$ per day. By monitoring serum values for sodium and chloride, throughout treatment, it is possible to state that continuous inhaled dry salt micro particles do not at all influence electrolyte balance.

Inhaled dry salt micro particles are already recommended, by the manufacturer, as treatment for asthma, chronic pulmonary obstructive disease, cystic fibrosis, acute and chronic bronchitis in both outpatient and hospital care.

The probable effect of the dry salt micro particles is that of lowering inflammatory edema, enhancing mucus mobility, quan-

ful extubation attempts.

The infants received between 12 and 30 days of continuous inhaled dry salt micro particles, in addition to the standard treatment for BPD and life support.

The inhaled dry salt micro particles were delivered directly into the infant's trachea, by connecting the salt cartridge to the mechanical ventilator circuit either in proximity to the endotracheal tube, tracheostomy cannula or along the inspiratory limb of the ventilation circuit (Figure 3). Air was pumped through the SaltMed cartridge via an electrical pump made available, in the SaltMed kit, by the manufacturer (figure 2, item 2). It is important to note that the electrical pump delivered an additional fraction of inspired oxygen $\left(\mathrm{FiO}_{2}\right)$ of $21 \%$ (atmospheric air) to each patient, $2.5 \mathrm{l} /$ min. Ventilators were set at $5 \mathrm{l} / \mathrm{min}$. Thus all calculations of patient delivered $\mathrm{FiO}_{2}$, while delivering dry salt micro particles, in this paper will be a median between the $\mathrm{FiO}_{2}$ delivered by the mechanical ventilator, as set by the physician and an addition-

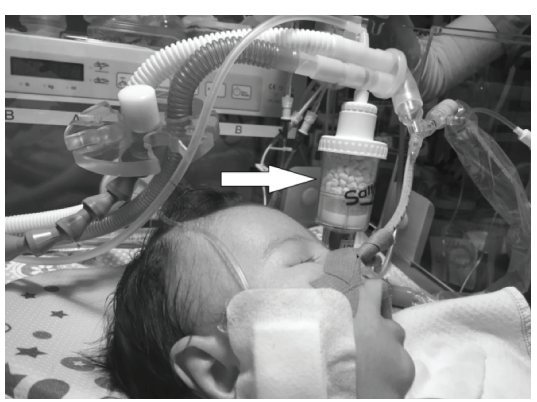

Figure 3. SaltMed cartridge connected to inhale part of mechanical ventilation circuit-marked by arrow.

al $\mathrm{FiO}_{2}$ of $21 \%$ delivered by the air pump. Connecting the Salt Med device decreased the $\mathrm{FiO}_{2}$ delivered to the patient, at the same settings of the ventilator and without changing the pressures measured.

Each patient had a set of cartridges assigned for individual use. The cartridges were changed every 12 hours for optimal delivery of dry salt micro particles. The same protocol was followed for each patient. When available, flow sensors were also attached to the ventilator circuit.

We measured peak inspiratory pressure (PIP), mean airway pressure (MAP), $\mathrm{FiO}_{2}$, tidal volumes (VT) and oxygenation index (OI) before and immediately after connecting the salt cartridge to the patients ventilation circuit and also every day during treatment. We also monitored serum values for sodium $(\mathrm{Na})$ and chloride $(\mathrm{Cl})$ in order to verify the impact of the dry salt micro particles on the serum electrolyte balance.

\section{CASE SERIES REPORT}

Patient \#1 was a 25-day old male neonate, born at 34 weeks gestation, birth weight 2340 g, Apgar score 6/7/8. He was admitted to our ward for treatment of a stage 4 intraventricular hemorrhage (IVH) with secondary hydrocephalus, hypoxicischemic encephalopathy and meningitis, heart failure, pulmonary hypertension and renal failure. During hospitalization he was diagnosed with severe BPD. During hospitalization he also acquired two nosocomial infections. The infant was intubated and mechanically ventilated on admission. He received inotropes, diuretics, antibiotics and underwent external ventricular drainage and insertion of an internal ventriculo-peritoneal shunt. Between 28-56 days postnatal age, he had several failed extubation attempts. At four months postnatal age a tracheostomy was performed with simultaneous administration of con- 
tinuous inhaled dry salt micro particles for a period of 30 days. Before and during the dry salt micro particles therapy, he also received intravenous aminophylline, 4 times a day. We measured and monitored $\mathrm{FiO}_{2}$, MAP and OI during all 30 days of dry salt therapy (Figure 4). Even though we administered a lower concentration of oxygen (two times $\mathrm{FiO}_{2}$ set vent + one time $21 \%$ divided by 3 ) immediately after administration of dry salt particles into the inspiratory limb of the ventilator circuit, the patient reached $100 \%$ saturation, so, we started to decrease $\mathrm{FiO}_{2}$ from $60 \%$ to $45 \%$ on the first day and to $30 \%$ on the second day. $\mathrm{FiO}_{2}$ was kept at this level until extubation. MAP did not change very much during this period. Oxygen Index was lowered significantly on the first two days and was kept around 8-10 until extubation. The patient was subsequently weaned off the ventilator after 7 months of mechanical ventilation. In addition, we monitored serum sodium and chloride values, and found no significant impact on electrolyte balance (Figure 5).

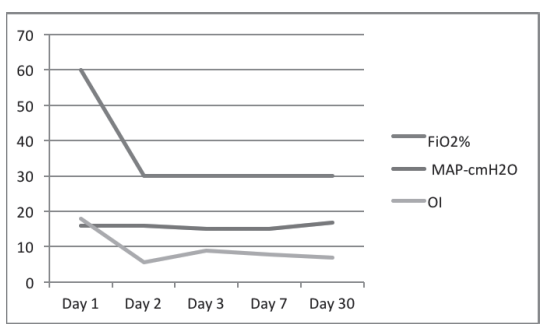

Figure 4. Patient \#1. $\mathrm{FiO}_{2} 21-100 \%$.

$\mathrm{FiO}_{2}$, fraction of inspired oxygen; MAP, mean airway pressure; OI, oxygenation index.

\begin{tabular}{llllll}
\hline Patient 1 & Day 1 & Day 2 & Day 3 & Day 7 & Day 30 \\
\hline $\mathrm{Na} \mathrm{mmol} / \mathrm{l}$ & 135.7 & 144.7 & 136.9 & 133.3 & 134.8 \\
\hline $\mathrm{Cl} \mathrm{mmol} / \mathrm{l}$ & 98 & 97 & 94 & 96 & 97
\end{tabular}

Figure 5. Serum $\mathrm{Na}$ and $\mathrm{Cl}$ values for patient 1.

Patient \#2 was a male neonate, born at 30 weeks gestation, birth weight $860 \mathrm{~g}$, Apgar score $3 / 5 / 7$, admitted to our NICU at 8 hours of life. The mother developed chorioamniotitis prior to giving birth. He was intubated and mechanically ventilated in the delivery room. After admission to our NICU he was diagnosed with mild to severe respiratory distress syndrome (RDS) and early onset neonatal sepsis (he received appropriate antibiotics, mild mechanical ventilation on high frequency oxygenation ventilation-HFOV, surfactant). After 28 days he was extubated and ventilated in non invasive modes for 2 months. For worsening $\mathrm{x}$ rays and work of breathing (WOB) he was ventilated again (HFOV). At 36 weeks postmenstrual age he was assessed and diagnosed with severe BPD. After 2 failed extubations, the patient was put back on continuous invasive respiratory support. Inhaled dry salt therapy was added as a complementary therapeutic measure for 30 days. On the day that dry salt micro particle therapy was introduced the patient was in a mediocre state. Even though the patient made a mild recovery, during the next several weeks he began to slowly deteriorate, becoming critical by the last day of dry salt micro particle therapy. He also received inhaled nitric oxide (iNO), milrinone and prostaglandin, with improvement in general status. After 5 months, the patient died due to severe metabolic acidosis and irreversible cardiopulmonary complications.

During dry salt particle therapy we measured and monitored $\mathrm{FiO}_{2}$, MAP, tidal volumes (VT), $\mathrm{SpO}_{2}$ and OI (Figure 6). We monitored serum sodium and chloride values, and found no significant impact on electrolyte balance (Figure 7).

Data collected revealed instant improvement in expiratory tidal volume (TVe). At the moment of introducing dry salt particles, TVe was $13 \mathrm{ml} /$ minute at time zero and rose to $17 \mathrm{ml} /$ minute after only ten minutes of inhaled dry salt micro particles having the same ventilator settings. After 21 days the patient developed pulmonary hypertension so nitrous oxide was also added to the patient's treatment regimen, because of ongoing pulmonary function deterioration due to BPD and pulmonary hypertension.

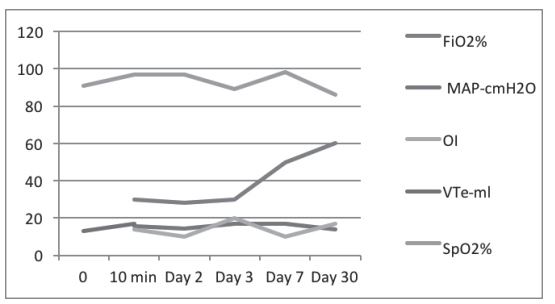

Figure 6. Patient \#2. $\mathrm{FiO}_{2} 21-100 \%$.

$\mathrm{FiO}_{2}$, fraction of inspired oxygen; MAP, mean airway pressure; OI, oxygenation index; VT, volume tidal.

\begin{tabular}{llllll}
\hline Patient 2 & Day 1 & Day 2 & Day 3 & Day 7 & Day 30 \\
\hline $\mathrm{Na} \mathrm{mmol} / \mathrm{l}$ & 136.2 & 134.3 & 133.1 & 143.2 & 143.8 \\
\hline $\mathrm{Cl} \mathrm{mmol} / \mathrm{l}$ & 99 & 97 & 99 & 106 & 122
\end{tabular}

Figure 7. Serum $\mathrm{Na}$ and $\mathrm{Cl}$ values for patient 2.
Patient \#3, a 38-day, term, male infant, birth weight 3300 g, Apgar score 9, diagnosed after birth with esophageal atresia, and operated on within the first days of life. The infant was referred to our hospital because of intense labored breathing with low $\mathrm{SpO}_{2}$ after postsurgery extubation. Upon admission he was intubated and mechanically ventilated until discharge. After further investigations he was diagnosed with severe tracheal stenosis. A tracheostomy was performed. Between 28 and 56 days postnatal age he was evaluated and diagnosed with severe BPD. He received a 15 day course of treatment with inhaled dry salt micro particles. On the day this therapy was initiated the patient was in a mediocre state. Other than mechanical ventilation no other medication, that could have impacted pulmonary function, was added to the patient's plan treatment while receiving inhaled dry salt micro particles. After discharge the patient was referred to another clinic for surgical treatment of the tracheal stenosis. We measured and monitored $\mathrm{FiO} 2, \mathrm{MAP}$, and OI during the days of dry salt therapy (figure 8). We monitored serum sodium and chloride values, and found no significant impact on electrolyte balance (Figure 9). We were able to drop $\mathrm{FiO} 2$ and maintain MAP at the same value until discharge.

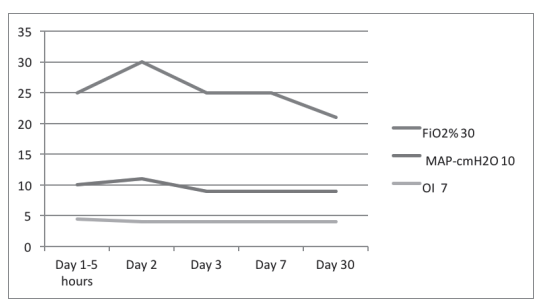

Figure 8. Patient \#3. $\mathrm{FiO}_{2} 21-100 \%$.

$\mathrm{FiO}_{2}$, fraction of inspired oxygen; MAP, mean airway pressure; OI, oxygenation index.

\begin{tabular}{llllll}
\hline Patient 3 & Day 1 & Day 2 & Day 3 & Day 7 & Day 15 \\
\hline $\mathrm{Na} \mathrm{mmol} / \mathrm{l}$ & 141 & 140.6 & 138 & 138.5 & 143.9 \\
\hline $\mathrm{Cl} \mathrm{mmol} / 1$ & 107 & 104 & 104 & 109 & 109 \\
\hline
\end{tabular}

Figure 9. Serum $\mathrm{Na}$ and $\mathrm{Cl}$ values for patient 3

Patient \#4 was a female neonate, born at 27 weeks gestation, birth weight $900 \mathrm{~g}$, Apgar score 8/9. She was intubated and mechanically ventilated shortly after birth. The neonate was admitted to out unit at the postnatal age of 68 days. She was diagnosed with IVH stage 4, secondary hydrocephalus, cerebral abscess and men- 
ingitis. Shortly after being admitted, she developed necrotizing enterocolitis with multiple bowel perforations, for which she underwent surgery. She was treated classically during hospitalization. At 36 weeks postmenstrual age she was diagnosed with severe BPD. After one failed attempt at extubation, the neonate received a 12 day course of inhaled dry salt micro particles. During this treatment no other medication or medical intervention was performed that could have impacted pulmonary function. The patient remained stable with no extraordinary events occurring. After 12 days of treatment we were able to successfully extubate the patient. Throughout these 12 days we were able to lower $\mathrm{FiO}_{2}$ to a minimum of $21 \%$ and to stabilize MAP. We measured and monitored $\mathrm{FiO}_{2}$, MAP, and OI during dry salt therapy (Figure 10). We monitored serum sodium and chloride values, and found no significant impact on electrolyte balance (Figure 11).

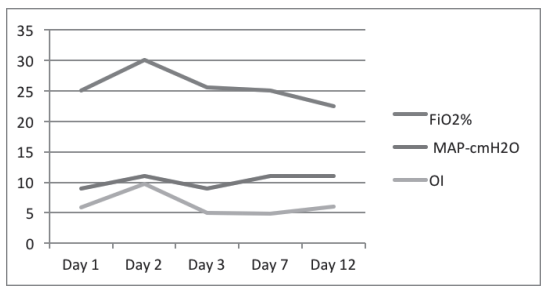

Figure 10. Patient \#4. $\mathrm{FiO}_{2} 21-100 \%$.

FiO2, fraction of inspired oxygen; MAP, mean airway pressure; OI, oxygenation index.

\begin{tabular}{llllll}
\hline Patient 4 & Day 1 & Day 2 & Day 3 & Day 7 & Day 12 \\
\hline $\mathrm{Na} \mathrm{mmol} / \mathrm{l}$ & 139.6 & 145.7 & 135.9 & 140.1 & 140.2 \\
\hline $\mathrm{Cl} \mathrm{mmol} / \mathrm{l}$ & 104 & 100 & 100 & 103 & 105 \\
\hline
\end{tabular}

Figure 11. Serum $\mathrm{Na}$ and $\mathrm{Cl}$ values for patient 4.

Patient \#5, a male neonate that was transferred to our clinic shortly after birth. He was born at 35 weeks gestation, birth weight $1530 \mathrm{~g}$, Apgar score 7/8. The premature neonate was diagnosed with esophageal atresia and Down syndrome. Surgical treatment consisted of primary thoracoscopic anastomosis of the esophageal tips. Complications after surgery occurred, esophageal fistula and candida infection being the principal ones. The patient was ventilated for a long time, with pulmonary parenchymal and interstitial involvement. Postoperative extubation failed and mechanical ventilation was reinstated. As we presented above, during hospitalization he developed a systemic fungal infection and mediastinitis. A bronchoscopy, after two more failed attempts to wean, was performed. He was diagnosed with a moderate to severe form of stenosis of the right main bronchus. A tracheostomy was performed. Between 28 and 56 days postnatal age he was diagnosed with severe BPD. We started administering inhaled dry salt micro particles and continued for 30 days. The patient was stable and remained so until the last day of dry salt therapy. Immediate improvement of tidal volume occurred. Initial value was measured at 30 $\mathrm{ml} / \mathrm{minute}$ at time zero and increased to 40 $\mathrm{ml} /$ minute after 10 minutes of inhaled dry salt micro particles. We monitored $\mathrm{FiO}_{2}$, MAP, tidal volume and OI during dry salt therapy (Figure 12). We monitored serum sodium and chloride values, and found no significant impact on electrolyte balance (Figure 13).

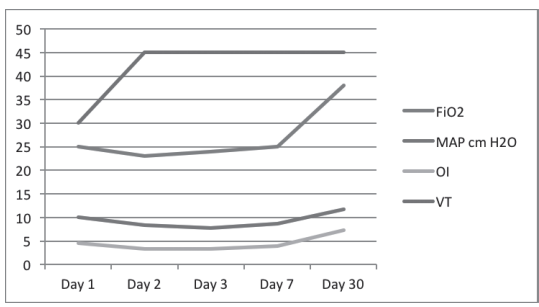

Figure 12. Patient \#5. $\mathrm{FiO}_{2} 21-100 \%$.

$\mathrm{FiO} 2$, fraction of inspired oxygen; MAP, mean airway pressure; OI, oxygenation index; VT, volume tidal.

\begin{tabular}{llllll}
\hline Patient 5 & Day 1 & Day 2 & Day 3 & Day 7 & Day 30 \\
\hline $\mathrm{Na} \mathrm{mmol} / \mathrm{l}$ & 134.9 & 137.6 & 135 & 135.1 & 134.2 \\
\hline $\mathrm{Cl} \mathrm{mmol} / \mathrm{l}$ & 107 & 107 & 106 & 102 & 104 \\
\hline
\end{tabular}

Figure 13. Serum $\mathrm{Na}$ and $\mathrm{Cl}$ values for patient 5.

\section{RESULTS}

We administered continuous inhaled dry salt micro particles to five patients diagnosed with severe BPD over a period of 12-30 days. After only 24 hours of therapy, in some cases sooner, we were able to lower $\mathrm{FiO}_{2}, \mathrm{MAP}$ and PIP to ventilate the patients, and recorded higher tidal volumes. All patients had been mechanically ventilated in conventional modes. Improved $\mathrm{SpO} 2$ was also seen. Throughout the inhaled dry salt micro particle therapy period our patients had better oxygenation indices. We were able to successfully extubate one of the patients after 12 days of continuous inhaled dry salt micro particles. In three out of five cases we succeeded in ventilating patients with atmospheric air and minimum pressures. They remained dependent on mechanical ventilation due to tracheal and bronchial illness (severe tracheomalacia, severe tracheal stenosis and moderate to severe right main bronchial stenosis). One of the patients deceased due to severe complications of underlying chronic pulmonary disease. Even in this case, we observed a brief but significant improvement in pulmonary function, which was not possible to obtain with other measures.

A larger quantity of secretions from the lower and upper respiratory tract were noted in all patients, as was to be expected considering the known mechanism of action of the dry salt micro particles on the respiratory epithelium. The increase in airway secretions was at its peak during the second and third days of therapy, started to decline by the seventh day and was resolved with fairly frequent aspirations. After daily monitoring of serum sodium and chloride levels, we concluded that continuous inhaled dry salt micro particles did not affect serum electrolyte balance, in any of our patients.

Two out of the five patients included in this report, received, simultaneously with the dry salt micro particles, medication that could have had influenced pulmonary function. Patient 1 was already undergoing treatment with Aminophylline, when dry salt micro particle therapy was introduced. No augmentation of dosage or administration schedule occurred. In the case of the second patient, because of progressive deteriorating pulmonary function and onset of pulmonary hypertension, it was decided to introduce nitric oxide, with a starting dose of $20 \mathrm{ppm}$. Dry salt particles brought about an advantage in the treatment of pulmonary function in this patient during both periods of treatment, before iNO and simultaneously with iNO. We cannot speculate a connection between worsening pulmonary hypertension and administration of the dry salt particles. The other three patients received no other medication or therapeutic intervention that could have improved pulmonary outcome.

\section{DISCUSSION}

BPD continues to be one of the leading causes of medium and long term morbidity and mortality in both premature and term neonates. For those who are discharged home, an often lengthy period of recurrent acute respiratory infections lies ahead, due to bronchial hyperreactivity and ongoing, 
lengthy pulmonary healing. These patients require prolonged hospitalization, resource-intensive care and costly treatment plans. The prospect of having a safe, easy to administer and cost effective means of reducing all of these complications related to BPD is of high interest. Inhaled dry salt micro particles appear to have an immediate effect on lung parameters, although there is no published data to support these findings. The exact mechanism of action for these immediate augmentations in respiratory parameters is not yet fully known.

\section{CONCLUSION}

Preliminary results strongly suggest that inhaled dry salt micro particles could be a safe, easy to administer and effective complementary medical intervention in the management of neonatal chronic lung disease, with a possible favorable effect on the respiratory and overall morbidity and mortality of infants with BPD. It is our wish to continue the study of this therapy and its effects on patients with BPD, in a more randomized and controlled manner.

\section{REFERENCES}

1. Stark AR, Redding G. Pathogenesis and clinical features of bronchopulmonary dysplasia. UpToDate, Wolters Kluwer

2. Mourani PM, Abman SH. Pulmonary vascular disease in bronchopulmonary dysplasia: pulmonary hypertension and beyond. Curr Opin Pediatr 2013 Jun;25(3):329-37.

3. Stoll BJ, Hansen NI, Bell EF, Shankaran S, Laptook AR, Walsh MC, et al. National Institute of Child Health and Human Development Neonatal Research Network. Neonatal outcomes of extremely preterm infants from the NICHD Neonatal Research Network.

4. Pediatrics 2010;126(3):443.

5. Walsh MC, Szefler S, Davis J, Allen M, Van Marter L, Abman S, et al.

6. Summary proceedings from the bronchopulmonary dysplasia group. Pediatrics 2006;117(3 Pt 2):S52.

7. Ann R Stark, Richard Martin, Gregory Redding. Prevention of bronchopulmonary dysplasia, UpToDate, Wolters Kluwer

8. Ann R Stark, Richard Martin, Gregory Redding. Management of bronchopulmonary dysplasia, UpToDate, Wolters Kluwer

9. Bancalari E, Wilson-Costello D, Iben SC. Management of infants with bronchopulmonary dysplasi in Nort America. Early Hum Dev 2005;81(2):171-9.

10. Shinwell ES, Portnov I, Meerpohl J, Karen T, Bassler D. Use of inhaled costicosteroids for the prevention and/orbronchopulmonary dysplasia in preterm infants: asystematic review protocol. Sys Rev 2015 Sept 25;4(1):127.doi:10.1186/s 13643-015-0108-1

11. Abman SH, Groothius JR. Pathophysiology and treatment of bronchopulmonary dysplasia. Pediatr Clin Nort Am 1994;41(2):277315.

12. Jasmine Shani Pharma D, Stephanie Phelps Pharma D. Nebulized Furosemide in the treatment of bronchopulmonary dysplasia in preterm infants. J Peditr Pharmacol Ther 2011;16(1):14-22.

13. Stelmach I, Jerzynska J, Bobrowska M, Brzozowska A, Majak P, Kuna P. The effect of inhaled heparin on airway responsiveness to metacholine in asthmatic children. Pol Arch Med Wewn 2001 Jul :106(1);567-72.

14. Gandia F, Rouatbi S, Lotiri I, Guenard H, Tabka Z. Inhaled magnesium floride reverse bronchospasma. J Smooth Muscle Res 2010;46(3):157-64.

15.Zhao W, Rouatbi S, Tabka Z, Guenard H. Inhaled sodium floride decreses airway resposiveness to acetylcholine analogs in vivo. Respir Physiol Neurobiol 2002 Aug 1;131(3):245-53. 